\title{
Parameter Estimation for Traffic Noise Models Using a Harmony Search Algorithm
}

\author{
Deok-Soon An, ${ }^{1}$ Young-Chan Suh, ${ }^{2}$ Sungho Mun, ${ }^{3}$ and Byung-Sik Ohm ${ }^{1}$ \\ ${ }^{1}$ Highway Research Division, SOC Research Institute, Korea Institute of Construction Technology, Goyang-si, \\ Gyeonggi-do 411-712, Republic of Korea \\ ${ }^{2}$ Department of Transportation \& Logistics Engineering, Hanyang University, Ansan-si, Gyeonggi-do 426-791, Republic of Korea \\ ${ }^{3}$ Department of Civil Engineering, Seoul National University of Science \& Technology, Seoul 139-743, Republic of Korea
}

Correspondence should be addressed to Sungho Mun; smun@seoultech.ac.kr

Received 28 June 2013; Revised 22 September 2013; Accepted 22 September 2013

Academic Editor: Zong Woo Geem

Copyright (C) 2013 Deok-Soon An et al. This is an open access article distributed under the Creative Commons Attribution License, which permits unrestricted use, distribution, and reproduction in any medium, provided the original work is properly cited.

\begin{abstract}
A technique has been developed for predicting road traffic noise for environmental assessment, taking into account traffic volume as well as road surface conditions. The ASJ model (ASJ Prediction Model for Road Traffic Noise, 1999), which is based on the sound power level of the noise emitted by the interaction between the road surface and tires, employs regression models for two road surface types: dense-graded asphalt (DGA) and permeable asphalt (PA). However, these models are not applicable to other types of road surfaces. Accordingly, this paper introduces a parameter estimation procedure for ASJ-based noise prediction models, utilizing a harmony search (HS) algorithm. Traffic noise measurement data for four different vehicle types were used in the algorithm to determine the regression parameters for several road surface types. The parameters of the traffic noise prediction models were evaluated using another measurement set, and good agreement was observed between the predicted and measured sound power levels.
\end{abstract}

\section{Introduction}

It is important to evaluate the primary impact of traffic noise for several different road surfaces in order to predict the interactive noise between road surfaces and vehicle tires. A number of noise prediction models [1-6] have been developed for environmental estimation of traffic noise levels in terms of vehicle and pavement types. For example, the vehicle types in the ASJ model $[1,2]$ are large vehicles, medium vehicles, light trucks, and cars, and the pavement types are dense-graded asphalt (DGA) and permeable asphalt (PA). Thus, the ASJ traffic noise prediction model is restricted to four vehicle types, varying vehicle speeds, and two pavement types.

In order to expand the applicability of the ASJ model, the present paper introduces a parameter estimation procedure based on a harmony search (HS) algorithm as follows: (a) the traffic noise for a targeted road surface is measured, (b) the parameters of the noise prediction model are estimated via the HS algorithm, and (c) the resulting coefficients are evaluated using another set of measurements (consisting of vehicle speeds and vehicle types) for a different traffic volume on the targeted road surface. To validate the proposed traffic noise prediction technique, traffic noise measurement sets from three different surface types were used in this study: stone mastic asphalt surfaces (SMA), $30 \mathrm{~mm}$ transversely tined Portland cement concrete surfaces ( $30 \mathrm{~mm}$ trans.), and $18 \mathrm{~mm}$ longitudinally tined Portland cement concrete surfaces (18 mm long.), as shown in Figure 1. The measurement site is a $7.7 \mathrm{~km}$ long. 2-lane highway along the side of the south bound of Jungbu inland highway in South Korea. This measurement section includes both asphalt and Portland cement concrete pavements.

This paper is organized as follows. Section 2 describes the ASJ model and vehicle characterization. Section 3 explains the application of the HS algorithm to estimate the parameters of ASJ-based noise prediction models, and Section 4 presents the conclusions of this research. 


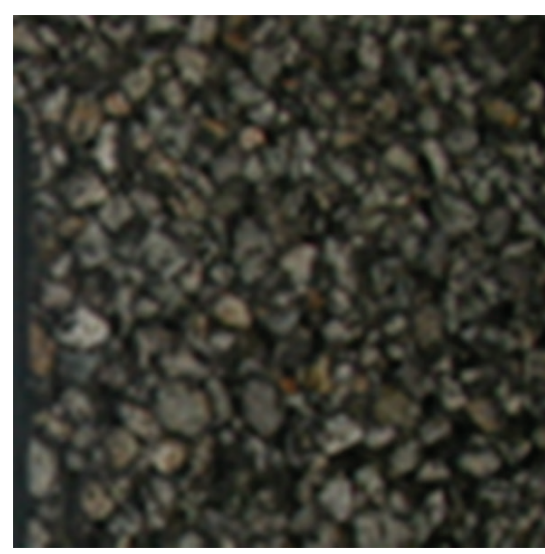

(a)

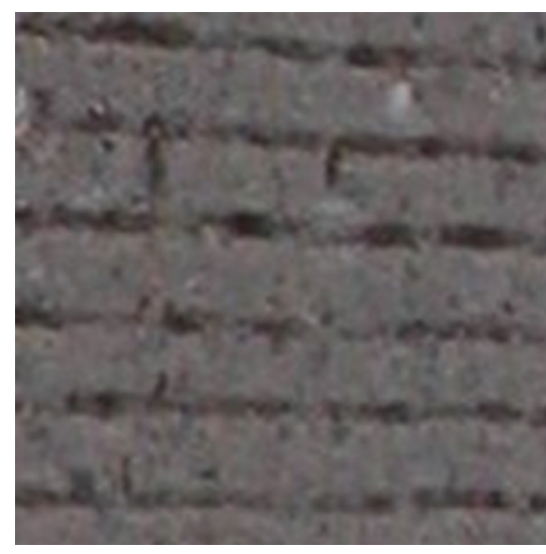

(b)

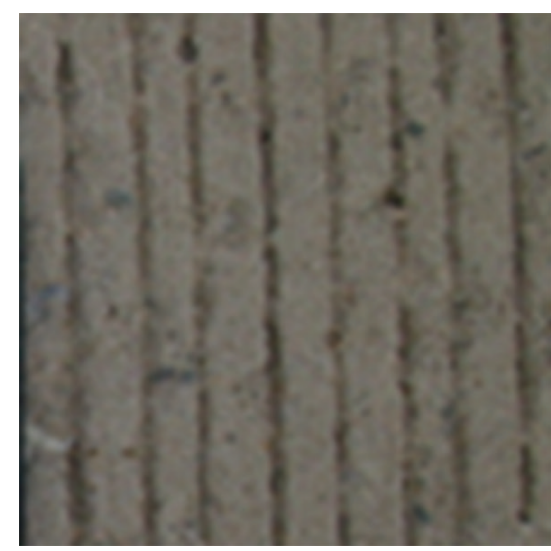

(c)

Figure 1: Pavement surface types: (a) SMA, (b) $30 \mathrm{~mm}$ trans., and (c) $18 \mathrm{~mm}$ long.

\section{ASJ Model}

The Acoustic Society of Japan (ASJ) published the ASJ model $[1,2]$ for calculating road traffic noise. The procedure involves calculating the noise level generated by traffic as well as the attenuation during noise propagation. The octave-band power spectrum for nominal midband frequencies $(63 \mathrm{~Hz}$ to $8 \mathrm{kHz}$ ) can be generated according to the ISO 9613-2 standard [7]. The ASJ model classifies vehicles into four types listed in Table 1.

The A-weighted overall sound power levels $\left(L_{\mathrm{WA}}\right)$ of the noise emitted by interactions between vehicles and pavement are listed in Table 2 for a dense-graded asphalt (DGA) surface. In terms of nominal midband frequencies, the individual Aweighted sound power level for each octave band $\left(L_{\mathrm{WA}, i}\right)$ is calculated as follows:

$$
L_{\mathrm{WA}, i}=L_{\mathrm{WA}}+\Delta L_{i}+\Delta L_{A, i}+\Delta L_{\mathrm{adj}}
$$

where $L_{\mathrm{WA}}$ is the overall sound power level $(\mathrm{dB})$ and $\Delta L_{i}$ is the relative level $(\mathrm{dB})$ at the $i$ th nominal mid-band frequency given by

$$
\Delta L_{i}=-10 \log _{10}\left\{1+\left(\frac{f_{i}}{2000}\right)^{2}\right\}-2.5 \log _{10}\left(\frac{f_{i}}{1000}\right)
$$

$\Delta L_{A, i}$ is a factor of the standard A-weighting frequency given by the following equation:

$$
\begin{aligned}
& \Delta L_{A, i}= 2.0+20 \log _{10} \\
& \times {\left[\left(12200^{2} \cdot f_{i}^{4}\right)\right.} \\
& \quad \times\left(\left(f_{i}^{2}+20.6^{2}\right)\left(f_{i}^{2}+12200^{2}\right)\right. \\
&\left.\left.\quad \times \sqrt{\left(f_{i}^{2}+107.7^{2}\right)\left(f_{i}^{2}+737.9^{2}\right)}\right)^{-1}\right],
\end{aligned}
$$

where $f_{i}$ is the nominal mid-band frequency. Finally, $\Delta L_{\text {adj }}$ is the correction factor defined by

$$
\Delta L_{\mathrm{adj}}=-10 \log _{10}\left\{\sum_{i=1}^{8} 10^{0.1\left(\Delta L_{i}+\Delta L_{A, i}\right)}\right\} .
$$

2.1. Equivalent Sound Power Level for a Road. The A-weighted sound power level emitted by a specific type of vehicle moving along a road over a specified time period, $L_{\text {WAT }}$, can be calculated via the following equation:

$$
L_{\mathrm{WAT}}=L_{\mathrm{WA}}+10 \log _{10}\left(\frac{3.6 \Delta l}{V} \cdot \frac{N}{3600}\right),
$$

where $L_{\mathrm{WA}}$ is the basic sound power level (dB) emitted by the vehicle (listed in Table 2 ), $\Delta l$ is the length of the road segment (in meters), $V$ is the mean speed for the vehicle type (in $\mathrm{km} / \mathrm{h}$ ), and $N$ is the hourly traffic flow for the vehicle type (in vehicles/h). The equivalent sound power level, $L_{\text {eq(WA), }}$ emitted by all vehicles moving along the road can then be calculated as follows:

$$
L_{\mathrm{eq}(\mathrm{WA})}=10 \log _{10}\left\{\sum_{i} 10^{L_{\mathrm{WAT}, i} / 10}\right\},
$$

where $L_{\mathrm{WAT}, i}$ is the A-weighted sound power level for each vehicle type. Here, $L_{\mathrm{WAT}, 1}, L_{\mathrm{WAT}, 2}, L_{\mathrm{WAT}, 3}$, and $L_{\mathrm{WAT}, 4}$ are the A-weighted sound power levels for a large vehicle, a medium vehicle, a light truck, and a car, respectively, as listed in Table 2. Furthermore, the attenuation (e.g., geometrical divergence, atmospheric absorption, ground effect, and screening structures) was calculated based on ISO 9613-2 [7].

\section{Application of the Harmony Search Algorithm}

3.1. Harmony Search Algorithm. This section describes the procedure for estimating the parameters of noise prediction models, using a harmony search (HS) algorithm that employs a heuristic algorithm based on an analogy with natural 
TABle 1: Vehicle types used in this study.

\begin{tabular}{lll}
\hline \multicolumn{1}{c}{ Type } & Specification \\
\hline Heavy vehicle & Large vehicle & $\begin{array}{l}\text { (i) Trucks equipped with three or more axles, which are } \\
\text { greater than } 8 \text { tons or above 5-ton cargo trailers } \\
\text { (ii) Buses providing } 30 \text { or more passengers } \\
\text { (iii) Large special purpose vehicles }\end{array}$ \\
Medium vehicle & $\begin{array}{l}\text { (i) Trucks equipped with two axles } \\
\text { (ii) Buses providing less than } 30 \text { passengers }\end{array}$ \\
\hline Light vehicle & Car & $\begin{array}{l}\text { Light trucks equipped with } 50 \text { to } 2000 \mathrm{cc} \text { engine } \\
\text { Car designed for the transportation of less than and equal to } \\
10 \text { passenger. }\end{array}$
\end{tabular}

TABLE 2: A-weighted sound power levels $\left(L_{\mathrm{WA}}\right)$ in $\mathrm{dB}$ for a densegraded asphalt (DGA) surface.

\begin{tabular}{lc}
\hline Vehicle types & Steady traffic flow $(40 \mathrm{~km} / \mathrm{h}$ to $140 \mathrm{~km} / \mathrm{h})$ \\
\hline Large vehicle & $54.4+30 \log _{10} V^{*}$ \\
Medium vehicle & $51.5+30 \log _{10} V$ \\
Light truck & $47.6+30 \log _{10} V$ \\
Car & $46.4+30 \log _{10} V$ \\
\hline
\end{tabular}

${ }^{*} V$ is a velocity.

phenomena [8-12]. The detailed procedure for applying a harmony search consists of four steps as follows.

(1) The algorithm parameters are specified. These include the harmony memory size (HMS), initialized as the number of solution vectors in the harmony memory (HM), the harmony memory consideration rate (HMCR, between 0 and 1 ), the pitch adjustment rate (PAR, between 0 and 1), and the maximum number of improvisations (or stopping criterion), which terminates the HS program. The optimization problem is specified as follows:

Minimize $f(X)$ subject to $x_{i} \in X_{i}=1,2, \ldots, N$,

where $f(X)$ is the objective function, $X$ is the set of decision variables $x_{i}, N$ is the number of decision variables, and $X_{i}$ is the possible range of values for the $i$ th decision variable; that is, $X_{L i} \leq X_{i} \leq X_{U i}$, where $X_{L i}$ and $X_{U i}$ are the respective lower and upper bounds for the $i$ th decision variable. To estimate the parameters of a noise prediction model, the following minimization function can be used:

$$
\begin{aligned}
& \text { Minimize }\left|L_{P_{-} \text {eq }(\mathrm{WA})}-L_{M_{-\mathrm{eq}}(\mathrm{WA})}\right| \\
& \text { subject to } x_{i} \in X_{i}=1,2, \ldots, N,
\end{aligned}
$$

where $L_{P_{-e q}(\mathrm{WA})}$ is the predicted equivalent sound power level of (5), which can be calculated as follows:

$$
\begin{aligned}
& L_{P_{\_} \mathrm{eq}(\mathrm{WA})} \\
& \quad=10 \log _{10}\left[10^{0.1\left\{L_{\mathrm{WAT}, 1}+L_{\mathrm{WAT}, 2}+L_{\mathrm{WAT}, 3}+L_{\mathrm{WAT}, 4}\right\}}\right],
\end{aligned}
$$

where $L_{\mathrm{WAT}, 1}, L_{\mathrm{WAT}, 2}, L_{\mathrm{WAT}, 3}$, and $L_{\mathrm{WAT}, 4}$ are the Aweighted sound power levels for a large vehicle, a medium vehicle, a light truck, and a car, respectively. $L_{M \_ \text {eq(WA) }}$ is the measured equivalent sound power level obtained from previous research [13, 14]. In this optimization problem, the A-weighted sound power levels can be defined as given in Table 3 . Thus, the coefficients of $x_{1}, x_{2}, x_{3}$, and $x_{4}$ must be determined via the HS algorithm. The slope is fixed in the ASJ models for both surface types (DGA and PA); therefore, the slope given in Table 3 is fixed at 30 .

(2) The HM matrix is initially filled with randomly generated solution vectors up to the HMS, together with the corresponding function values of the random vectors $f(X)$ :

$\mathrm{HM}=\left[\begin{array}{cccc|c}x_{1}^{1} & x_{2}^{1} & x_{3}^{1} & x_{4}^{1} & f\left(X^{1}\right) \\ x_{1}^{2} & x_{2}^{2} & x_{3}^{2} & x_{4}^{2} & f\left(X^{2}\right) \\ \vdots & \vdots & \cdots & \vdots & \vdots \\ x_{1}^{\mathrm{HMS}} & x_{2}^{\mathrm{HMS}} & x_{3}^{\mathrm{HMS}} & x_{4}^{\mathrm{HMS}} & f\left(X^{\mathrm{HMS}}\right)\end{array}\right]$.

(3) A column vector of the newly generated harmony, $X^{\prime}=\left(x_{1}^{\prime} x_{2}^{\prime} x_{3}^{\prime} x_{4}^{\prime}\right)$, is improvised utilizing the following three mechanisms: (a) random selection, (b) memory consideration, and (c) pitch adjustment. In the random selection, the value of each decision variable, $x_{i}{ }^{\prime}$, in the column vector is randomly chosen within the range of values with a probability of (1HMCR). HMCR (which is between 0 and 1 ) is the rate at which a single value is chosen from the historical values stored in the HM. The value of each decision variable selected by memory consideration is examined in terms of pitch adjustment. This operation uses the PAR parameter (which is the rate of the necessary pitch adjustment according to the neighboring pitches) with a probability of HMCR $\times$ 
TABLE 3: A-weighted sound power levels $\left(L_{\mathrm{WA}}\right)$ in $\mathrm{dB}$ for the different surface types.

\begin{tabular}{lc}
\hline Vehicle types & Steady traffic flow $(40 \mathrm{~km} / \mathrm{h}$ to $140 \mathrm{~km} / \mathrm{h})$ \\
\hline Large vehicle & $x_{1}+30 \log _{10} V^{*}$ \\
Medium vehicle & $x_{2}+30 \log _{10} V$ \\
Light truck & $x_{3}+30 \log _{10} V$ \\
Car & $x_{4}+30 \log _{10} V$ \\
\hline
\end{tabular}

${ }^{*} V$ is a velocity.

TABLE 4: Vehicle velocities and hourly traffic flows.

\begin{tabular}{lcc}
\hline Vehicle types & Average velocities & Hourly traffic flows \\
\hline Large vehicle & $90 \mathrm{~km} / \mathrm{h}$ & 48 vehicles $/ \mathrm{h}$ \\
Medium vehicle & $95 \mathrm{~km} / \mathrm{h}$ & 40 vehicles $/ \mathrm{h}$ \\
Light truck & $97 \mathrm{~km} / \mathrm{h}$ & 96 vehicles $/ \mathrm{h}$ \\
Car & $101 \mathrm{~km} / \mathrm{h}$ & 208 vehicles $/ \mathrm{h}$ \\
\hline
\end{tabular}

PAR. Pitch adjustment is applied to each variable as follows:

$$
x_{i}^{\prime} \longleftarrow\left\{\begin{array}{c}
x_{i}^{\prime}+\text { rand } \times b w, \\
\text { with a probability of HMCR } \times \text { PAR } \times 0.5 \\
x_{i}^{\prime}-\text { rand } \times b w, \\
\text { with a probability of } \mathrm{HMCR} \times \mathrm{PAR} \times 0.5 \\
x_{i}^{\prime}, \text { with a probability of } \mathrm{HMCR} \times(1-\mathrm{PAR}) .
\end{array}\right.
$$

If the newly generated column vector is better than the worst harmony in the HM, based on evaluation of the objective function, the newly generated column vector is included in the HM, and the existing worst harmony is excluded from the HM.

(4) If the stopping criterion (or maximum number of improvisations) is satisfied, the computation is terminated. Otherwise, Steps 3 and 4 are repeated.

\subsection{Application to Parameter Estimation for Noise Prediction} Models. In order to estimate the parameters of noise prediction models based on the objective function of (8), noise measurements for three different road surfaces were obtained from previous research, which was conducted on a test track $[13,14]$. The vehicle velocities and hourly traffic flows are listed in Table 4.

To apply the HS algorithm to parameter estimation for the noise prediction models, the four coefficients were determined for each road surface type (stone mastic asphalt (SMA) surface, $30 \mathrm{~mm}$ transversely tined Portland cement concrete surface (30 mm trans.), and $18-\mathrm{mm}$ longitudinally tined Portland cement concrete surface (18 $\mathrm{mm}$ long.)), as shown in Figure 2 and Table 5, based on the training data from Table 4 . In this way, the coefficients of the noise prediction models, which are dependent on the road surface type, can be updated via the HS algorithm. As a result, noise prediction models can be provided for various surface types by using the HS algorithm to update the ASJ model equations.

Another set of testing data (given in Table 6) was used to evaluate whether or not the updated noise prediction models

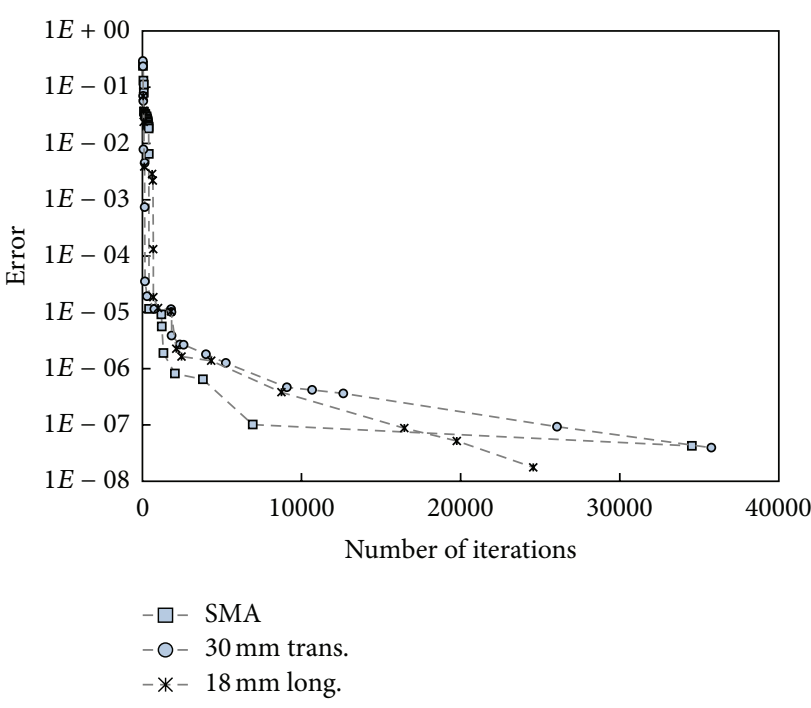

FIGURE 2: Minimizing the error function of (8) through the HS algorithm.

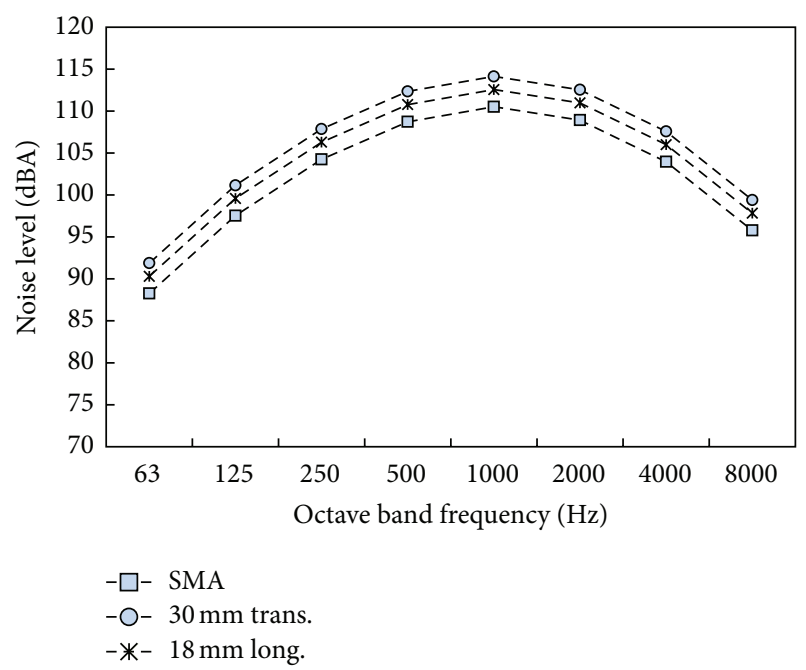

FIGURE 3: Octave band frequency versus noise level.

(with the four coefficients estimated by the HS algorithm) provided results consistent with measured noise levels. The predictions and measurements are compared in Table 7; good agreement was noted for all three surface types. Using the original ASJ model, the prediction value is resulted in a same noise level regardless of surface types and in bad agreement when compared with the measured noise levels.

Finally, the A-weighted sound power levels for the individual octave bands were estimated for the SMA, $30 \mathrm{~mm}$ trans., and $18 \mathrm{~mm}$ long. surface types, utilizing the parameters estimated via the HS algorithm and (1). For example, Figure 3 shows the A-weighted sound power levels for the octave bands for the three different surface types in the case of the large vehicle speeding at $80 \mathrm{~km} / \mathrm{h}$. 
TABLE 5: Determination of the model coefficients via the HS algorithm.

\begin{tabular}{lcccc}
\hline Surface types & Large vehicle $\left(x_{1}\right.$ in Table 3$)$ & Medium vehicle $\left(x_{2}\right.$ in Table 3$)$ & Light truck $\left(x_{3}\right.$ in Table 3) & Car $\left(x_{4}\right.$ in Table 3) \\
\hline SMA & 58.050 & 53.693 & 48.456 & 45.787 \\
30 mm trans. & 61.669 & 58.473 & 51.246 & 46.020 \\
18 mm long. & 60.097 & 54.428 & 50.095 & 45.360 \\
\hline
\end{tabular}

TABLE 6: Vehicle velocities and hourly traffic flows used as evaluation data.

\begin{tabular}{lcr}
\hline Vehicle types & Average velocities & Hourly traffic flows \\
\hline Large vehicle & $85 \mathrm{~km} / \mathrm{h}$ & $35 \mathrm{vehicles} / \mathrm{h}$ \\
Medium vehicle & $90 \mathrm{~km} / \mathrm{h}$ & $58 \mathrm{vehicles} / \mathrm{h}$ \\
Light truck & $95 \mathrm{~km} / \mathrm{h}$ & 87 vehicles/h \\
Car & $103 \mathrm{~km} / \mathrm{h}$ & $198 \mathrm{vehicles} / \mathrm{h}$ \\
\hline
\end{tabular}

TABLE 7: Comparison of predicted and measured traffic noise levels.

\begin{tabular}{lccc}
\hline Surface types & Predicted levels & Measured levels & ASJ model prediction \\
\hline SMA & $96.1 \mathrm{~dB}$ & $96.2 \mathrm{~dB}$ & $94.4 \mathrm{~dB}$ \\
$30 \mathrm{~mm}$ trans. & $99.5 \mathrm{~dB}$ & $99.7 \mathrm{~dB}$ & $94.4 \mathrm{~dB}$ \\
$18 \mathrm{~mm}$ long. & $97.3 \mathrm{~dB}$ & $97.6 \mathrm{~dB}$ & $94.4 \mathrm{~dB}$ \\
\hline
\end{tabular}

\section{Conclusions}

In this study, it was shown that the optimization problem related to updating the noise prediction models for several surface types could be solved using an HS algorithm process. The process involves (a) obtaining measurements for different road surfaces, (b) estimating the coefficients of the noise prediction models using this measurement set as training data, and (c) evaluating the estimated coefficients using another measurement set as testing data. When this procedure was utilized, an evaluation of the parameters of the traffic noise prediction model yielded good agreement between predicted and measured sound power levels.

\section{Conflict of Interests}

The authors of the paper do not have a direct financial relationship with the commercial identities mentioned in this paper.

\section{Acknowledgment}

This research was supported by a grant from a Strategic Research Project "Development of Traffic Noise Modeling and Noise Reduction Technology as Road Traffic Noise by Location" funded by the Korea Institute of Construction Technology.

\section{References}

[1] Acoustic Society of Japan, "ASJ prediction model for road traffic noise," Bull. Acoustic Society of Japan, vol. 55, no. 1, pp. 281-321, 1999.

[2] K. Yamamoto, "Road traffic noise prediction model "ASJ RTNModel 2008": report of the research committee on road traffic noise," Acoustical Science and Technology, vol. 31, no. 1, pp. 2-55, 2010.

[3] U. Sandberg, "Road traffic noise-the influence of the road surface and its characterization," Applied Acoustics, vol. 21, no. 2, pp. 97-118, 1987.

[4] C. Steele, "Critical review of some traffic noise prediction models," Applied Acoustics, vol. 62, no. 3, pp. 271-287, 2001.

[5] D. S. Cho, J. H. Kim, T. M. Choi, B. H. Kim, and D. Manvell, "Highway traffic noise prediction using method fully compliant with ISO 9613: comparison with measurements," Applied Acoustics, vol. 65, no. 9, pp. 883-892, 2004.

[6] T. Bennert, D. Hanson, A. Maher, and N. Vitillo, "Influence of pavement surface type on tire/pavement generated noise," Journal of Testing and Evaluation, vol. 33, no. 2, pp. 94-100, 2005.

[7] International Organization for Standardization, ISO 96132:1996. Acoustics-attenuation of sound during propagation outdoors-part 2: general method of calculation, 1996.

[8] S. Mun and Z. W. Geem, "Determination of viscoelastic and damage properties of hot mix asphalt concrete using a harmony search algorithm," Mechanics of Materials, vol. 41, no. 3, pp. 339353, 2009.

[9] S. Mun and Z. W. Geem, "Determination of individual sound power levels of noise sources using a harmony search algorithm," International Journal of Industrial Ergonomics, vol. 39, no. 2, pp. 366-370, 2009.

[10] Z. W. Geem, "Particle-swarm harmony search for water network design," Engineering Optimization, vol. 41, no. 4, pp. 297311, 2009.

[11] J. Fourie, R. Green, and Z. W. Geem, "Generalised adaptive harmony search: a comparative analysis of modern harmony search," Journal of Applied Mathematics, vol. 2013, Article ID 380985, 13 pages, 2013.

[12] S. Lee, S. Mun, and H. Moon, "Determination of pavement rehabilitation activities through a permutation algorithm," Journal of Applied Mathematics, vol. 2013, Article ID 252808, 5 pages, 2013.

[13] S. Mun, D.-S. Cho, and T.-M. Choi, "Influence of pavement surface noise: the Korea Highway Corporation test road," 
Canadian Journal of Civil Engineering, vol. 34, no. 7, pp. 809816, 2007.

[14] D.-S. Cho and S. Mun, "Determination of the sound power levels emitted by various vehicles using a novel testing method," Applied Acoustics, vol. 69, no. 3, pp. 185-195, 2008. 


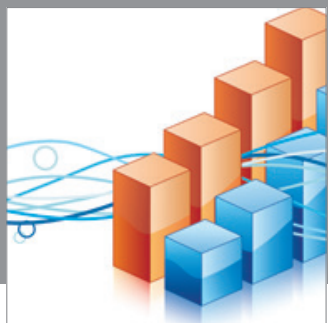

Advances in

Operations Research

mansans

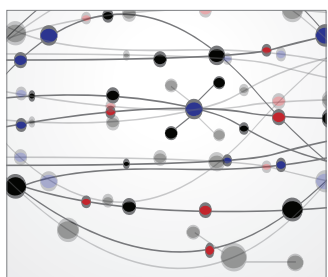

The Scientific World Journal
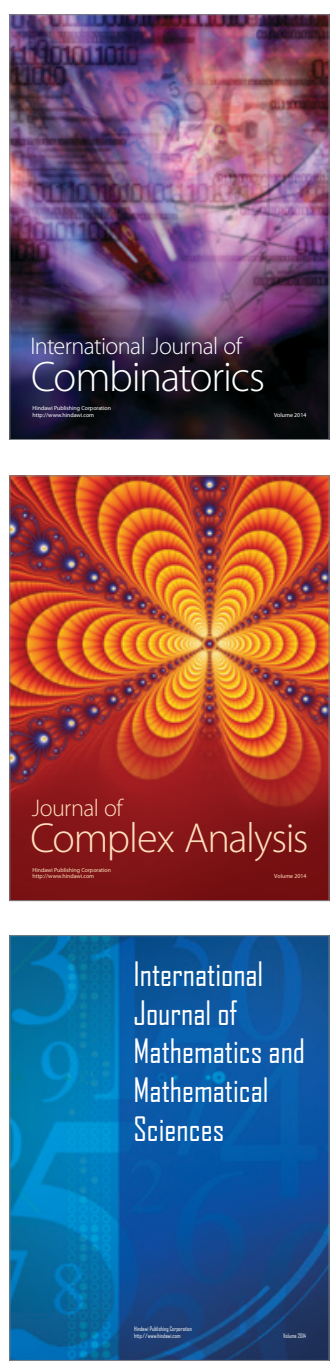
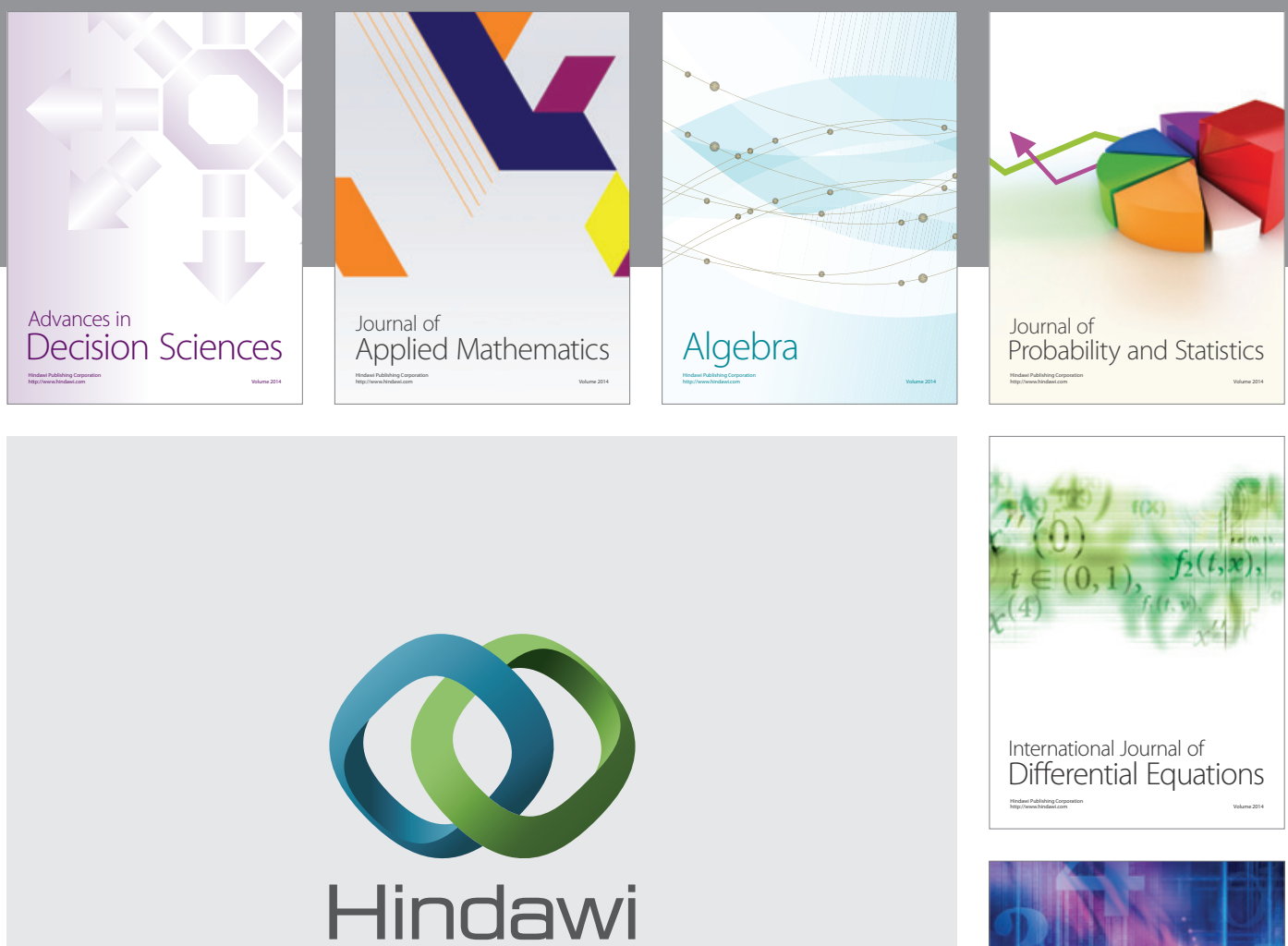

Submit your manuscripts at http://www.hindawi.com
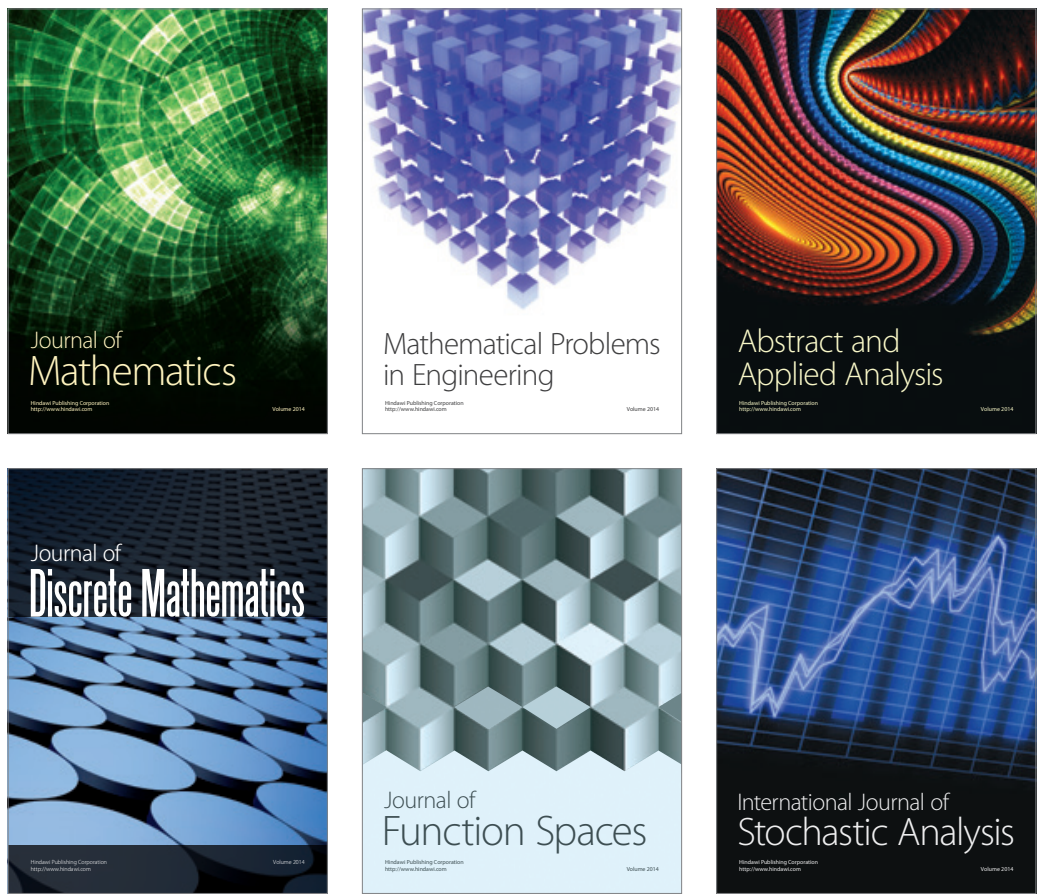

Journal of

Function Spaces

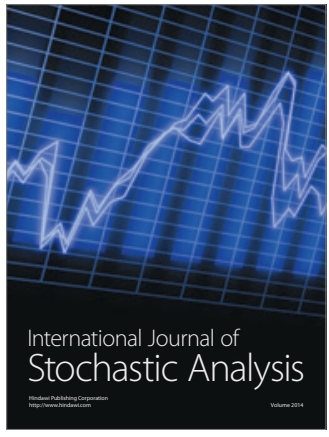

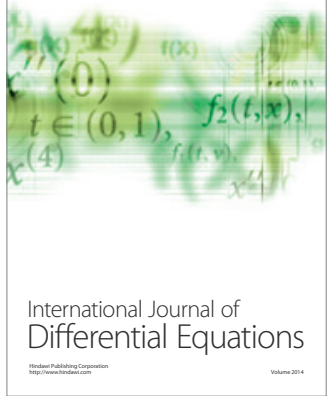
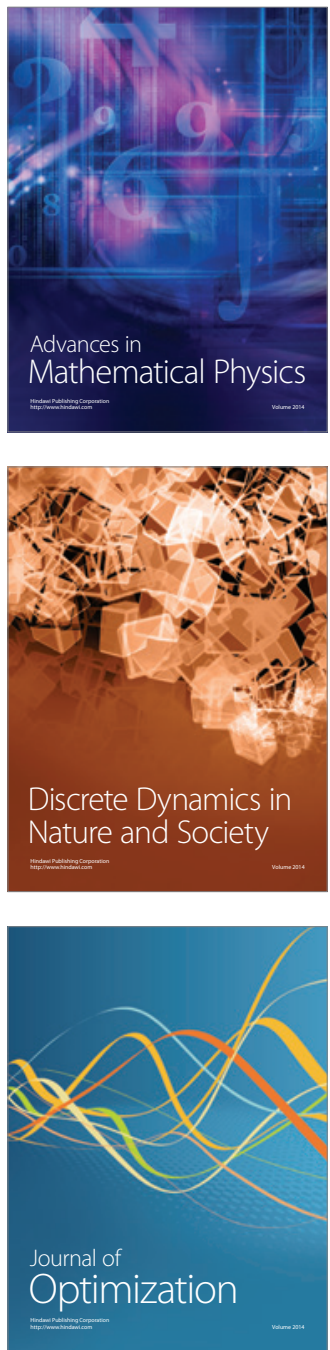